\title{
Soft tissue aneurysmal bone cyst in a 10-year-old girl
}

\author{
YONGQIANG HAO ${ }^{1}$, LEI WANG ${ }^{1}$, MENGNING YAN $^{1}$, FANGCHUN JIN $^{1}$, \\ SHENGFANG GE ${ }^{2}$ and KERONG DAI ${ }^{1}$ \\ ${ }^{1}$ Department of Orthopaedics Shanghai Key Laboratory of Orthopaedic Implant; ${ }^{2}$ Department of Ophthalmology, \\ Ninth People's Hospital, Shanghai Jiao Tong University School of Medicine, Shanghai 200011, P.R. China
}

Received October 3, 2011; Accepted December 12, 2011

DOI: $10.3892 / \mathrm{ol} .2011 .530$

\begin{abstract}
We report the case of a 10-year-old girl with a soft tissue aneurysmal bone cyst (STABC) located in the posterior aspect of the left shoulder. Conventional radiography revealed an oblong mass with a calcified rim. On the computed tomography scan, the lesion appeared to have a non-uniform intralesional density with an incomplete rim. Magnetic resonance imaging revealed a multi-cavity lesion with fluid-fluid levels. Following pathological examination, the lesion was diagnosed as a STABC. This may be only the twentieth reported case in the English literature of this extremely rare benign tumor occurring in soft tissue. Eight months after surgery the patient was assessed at our outpatient clinic and found to have excellent mobility of her left shoulder and no sign of recurrence.
\end{abstract}

\section{Introduction}

An aneurysmal bone cyst (ABC) is a benign, locally aggressive and expansile tumor that typically occurs in the long bones or vertebral bodies of children and young adults. Radiographically, a lytic lesion and expansion are the basic characteristics of $\mathrm{ABC}$, which usually has well-defined margins. Histologically, the tumor contains blood-filled cystic spaces separated by fibrous septa containing osteoclast-type giant cells, fibroblasts and reactive woven bone $(1,2)$.

Previously, ABC was believed to occur exclusively in bone (3), but in recent years a few cases of soft tissue $A B C$ (STABC) have been reported $(1,4-7)$. STABC is a recently recognized extremely rare tumor with fewer than 20 welldocumented cases in the literature (7), as shown in Table I. We report the imaging and pathological findings of a STABC in a 10-year-old girl and discuss its differential diagnosis.

Correspondence to: Professor Yongqiang Hao, Department of Orthopaedics, Shanghai Key Laboratory of Orthopaedic Implant, Ninth People's Hospital, Shanghai Jiao Tong University School of Medicine, 639 Zhizaoju Road, Shanghai 200011, P.R. China E-mail: hao_yongqiang@hotmail.com

Key words: aneurysmal bone cyst, soft-tissue, neoplasm, child, differential diagnosis

\section{Patient and methods}

The study was carried out according to the principles of the Declaration of Helsinki; informed consent was obtained and Shanghai Ninth People's Hospital Ethics Committee approved the study. The patient images contained in this article were captured by a hospital-based photographer at Shanghai Ninth People's Hospital, Shanghai Jiao Tong University School of Medicine (8). Permission to use these images in this study has been obtained from the parents of the girl who participated in this study.

A 10-year-old girl presented with a one-month history of pain in her left shoulder. She was admitted to hospital as the pain had increased over the previous 10 days, affecting the mobility of her left shoulder. Her parents denied any trauma to the area. Physical examination revealed a $6 \times 5-\mathrm{cm}$ painful lump in the posterior aspect of the left shoulder; the lump was solid, smooth and non-pulsatile. Shoulder mobility was limited; initiative outreach was $<80^{\circ}$, extension was $<30^{\circ}$, and anteflexion was $<30^{\circ}$. There were no signs of inflammation, and the white cell count was $5.8 \times 10^{9}$ cells $/ 1$. Serum electrolytes were normal.

Radiography indicated a round soft tissue lesion with a well-defined calcification margin located at the posterior aspect of the left shoulder, next to the scapula and proximal humerus. The adjacent bony structures were not involved, and there was no significant periosteal reaction on these bones (Fig. 1A). Computed tomography (CT) revealed an abnormality of the soft tissue in the left shoulder with an arcuated thin rim with an ambiguous density, suggesting calcification (Fig. 1B). The intralesional density was slightly uneven (Fig. 1C).

Magnetic resonance imaging (MRI) revealed a rectangular $5.9 \times 4.6-\mathrm{cm}$ soft tissue lesion in the posterior aspect of the left shoulder with an uneven intralesional signal. On T1-weighted MRI, the lesion showed an overall signal intensity (SI) similar to that of the surrounding normal muscles. The SI of the intralesional septa appeared a little higher than that of the separated areas (Fig. 2A). By contrast, the mass showed a predominantly high SI with a well-defined margin on T2-weighted MRI; the SI of the intralesional septa had a low to intermediate SI, while the separated areas were of high SI (Fig. 2B). Fluid-fluid levels were also observed in our patient (Fig. 2C). Following administration of gadopentetate dimeglumine, the lesion manifested peripheral and intralesional septa enhancement, while the separated areas were not significantly enhanced (Fig. 2D). 
A

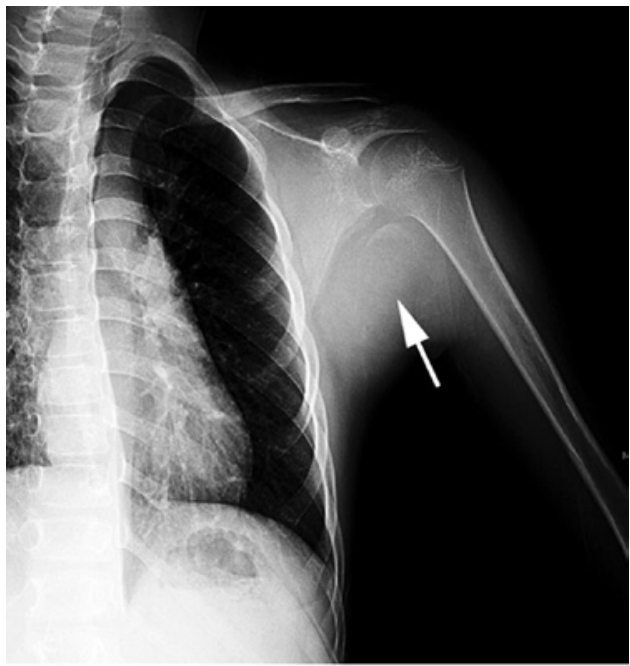

B

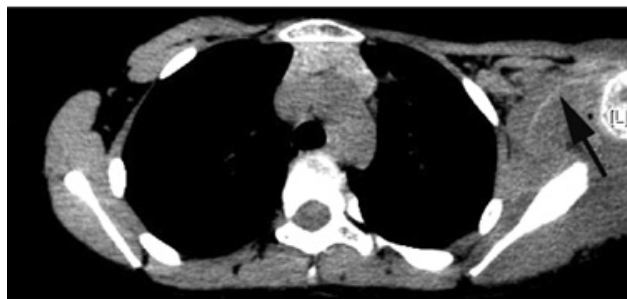

C

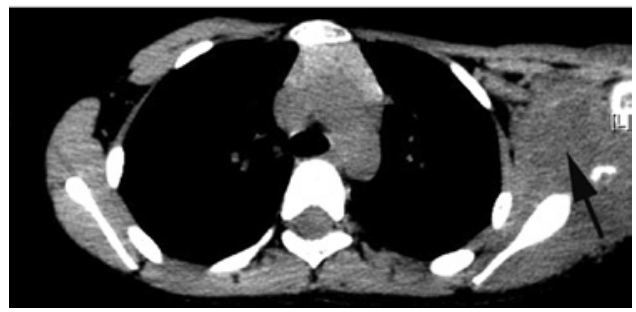

Figure 1. (A) Anteroposterior radiographs of the left shoulder showing a round soft tissue lesion with a well-defined calcified margin. (B and C) An axial CT scan shows a non-uniform soft tissue mass with an incomplete rim of osseous density. CT, computed tomography.

MRI further demonstrated that the scapula and proximal humerus were not involved and the sclerotin was normal.

The mass was subsequently completely excised under general anesthesia. Intraoperatively, we found that there was no relationship between the tumor and the adjacent bones and the mass was mainly located between the infraspinatus muscle, teres major muscle and tere muscle (Fig. 3A). On gross inspection, the specimen was a rectangular $8.8 \times 5.7 \times 4.6-\mathrm{cm}$ soft tissue mass (Fig. 3B), the exterior of which consisted of a rim formed partly of fibrous tissue with areas of a thin eggshell-like layer of bone (Fig. 3C). The center of the lesion consisted of irregular blood-filled cavities separated by septa of varying thickness (Fig. 3C). Microscopically, the lesion was composed of cystic blood-filled spaces. The cyst walls contained fibroblasts, inflammatory cells, multinucleated giant cells and reactive woven bones (Fig. 4).

\section{Discussion}

$\mathrm{ABC}$ is a type of benign lesion that may occur in any bone, but rarely in soft tissue. It occurs predominantly during the first two decades of life. An ABC is composed of numerous

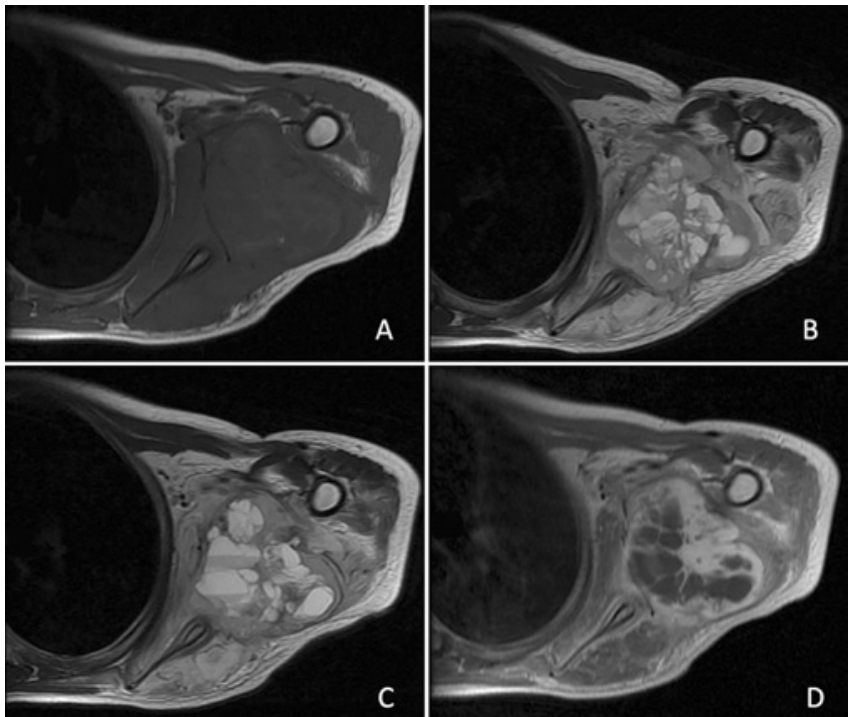

Figure 2. (A) By axial T1-weighted MRI, the SI of the lesion is identical to that of the surrounding muscles; the SI of intralesional septa appeared a little higher than the separated areas. (B) By axial T2-weighted MRI, the intralesional septa show low to intermediate SI, while the separated areas are of high SI. (C) By T2-weighted MRI, fluid-fluid levels are present. (D) Axial T1-weighted MRI following the administration of gadopentetate dimeglumine shows peripheral and intralesional septal enhancement. MRI, magnetic resonance imaging; SI, signal intensity.

irregular cysts filled with blood and separated by connective tissue septa containing fibroblasts, osteoclast-type giant cells and reactive bone $(1,2)$. It usually appears on radiographs as an eccentric lytic and expansile lesion with a well-defined margin within bones (1).

ABC was first described by Jaffe and Lichtenstein in 1942 (9). Its development has been widely regarded as a reactive process since then. However, it is recognized that there is no previous lesion in the majority of cases of this disease. Panoutsakopoulos et al (10) demonstrated the presence of a chromosomal translocation $\mathrm{t}(16 ; 17)(\mathrm{q} 22 ; \mathrm{p} 13)$ in two osseous cases, suggesting a neoplastic process. These initial findings were later confirmed by several subsequent cytogenetic studies $(11,12)$. Oliveira et al (13) reported that the $\mathrm{t}(16 ; 17)(\mathrm{q} 22 ; \mathrm{p} 13)$ translocation fuses the promoter region of the osteoblast cadherin 11 gene (CHD11) on chromosome 16q22 to the entire coding sequence of the ubiquitin protease USP6 gene. Ye et al (14) showed that the overexpression of USP6 in pre-osteoblastic MC3T3 cells is sufficient to drive the formation of tumors that reproduced the molecular and histological features of ABC. These authors also suggested that USP6 may play a direct role in establishing a degradative and vascularized microenvironment. Five partner genes known to upregulate USP6 expression in $\mathrm{ABC}$ have been identified: CDH11(16Q22), ZNF9(3Q21), OMD(9Q22), COL1A1(17Q21) and TRAP(1P34) (15). In addition, Geiersbach et al (16) reported an $\mathrm{ABC}$ case with an SS18 rearrangement, which has not previously been described. These findings clearly demonstrate that $\mathrm{ABC}$ is a neoplastic disease.

STABC is extremely rare. The number of published STABC cases does not exceed 20, with only a few epidemiological and histological reports (7). Of these cases, only 5 are in the pediatric age group, as shown in Table I. Our patient is 
Table I. Reported cases of soft-tissue aneurysmal bone cyst.

\begin{tabular}{|c|c|c|c|}
\hline Case & Age (years) & Site & Author and year \\
\hline 1 & 32 & Thigh & Salm, 1972 \\
\hline 2 & 45 & Abdominal wall & Salm, 1972 \\
\hline 3 & 15 & Groin & Amir, 1992 \\
\hline 4 & 7 & Left common carotid & Petrik, 1993 \\
\hline 5 & 20 & Infraspinous region & Rodriguez-Peralto, 1994 \\
\hline 6 & 57 & Left upper arm & Lopez-Barea, 1996 \\
\hline 7 & 29 & Left retroclavicular region & Shannon, 1997 \\
\hline 8 & 8 & Shoulder & Dal Cin, 2000 \\
\hline 9 & 51 & Pelvis & Samura, 2000 \\
\hline 10 & 8 & Right shoulder & Nielsen, 2002 \\
\hline 11 & 29 & Right groin & Nielsen, 2002 \\
\hline 12 & 37 & Upper arm & Nielsen, 2002 \\
\hline 13 & 28 & Left deltoid & Nielsen, 2002 \\
\hline 14 & 30 & Thigh & Nielsen, 2002 \\
\hline 15 & 21 & Right gluteus medius & Wang, 2004 \\
\hline 16 & 12 & Left thigh & Ajilogba, 2005 \\
\hline 17 & 10 & Left thigh & Ellison, 2007 \\
\hline 18 & 26 & Right thigh & Pietschmann, 2011 \\
\hline 19 & 38 & Left upper arm & Pietschmann, 2011 \\
\hline
\end{tabular}

The data of the top 15 cases come from reference 5 .
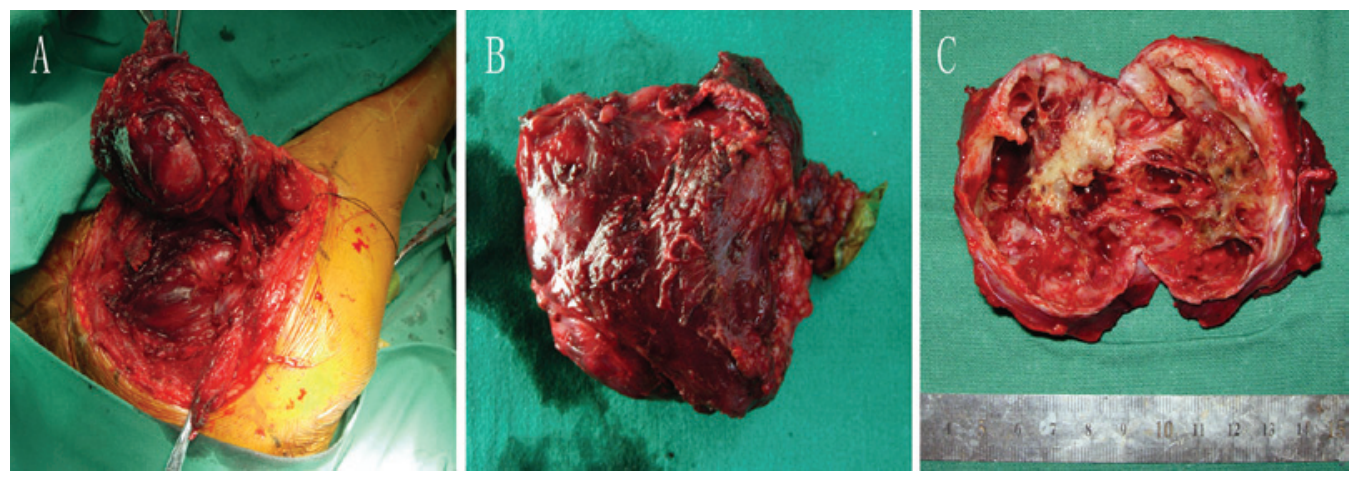

Figure 3. (A) The mass was mainly located between the infraspinatus muscle, the teres major muscle and the tere muscle without involving adjacent bones. (B) The general appearance of the tumor was a rectangular $8.8 \times 5.7 \times 4.6-\mathrm{cm}$ soft tissue mass. (C) Image showing a cross-section of the resected lesion. The lesion has a well-defined rim with areas of a thin eggshell-like layer of bone and is composed of blood-filled cavities separated by septa of various thicknesses.
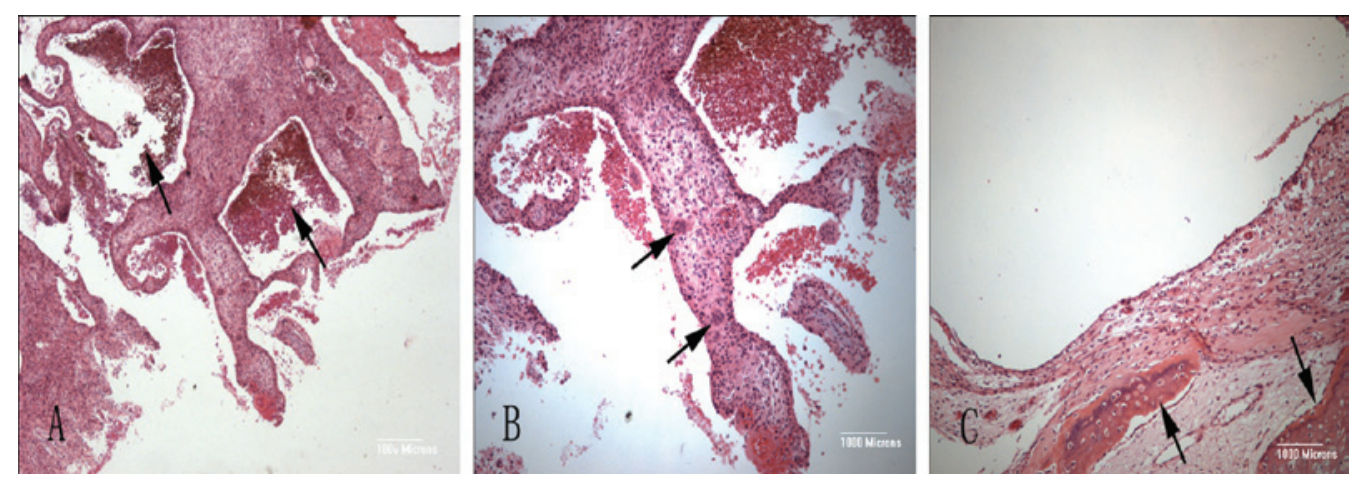

Figure 4. Histological features of STABC (hematoxylin and eosin). (A) Photomicrograph showing blood-filled cystic spaces (magnification, x50). (B) In the connective tissue septa, some multinucleated giant cells can be observed (magnification, x100). (C) Reactive woven bone can be observed in the fibrous septa of the STABC (magnification, x100). STABC, soft-tissue aneurysmal bone cyst. 
a 10-year-old girl with a lesion in the posterior aspect of the left shoulder. STABC shows a rather similar appearance to its osseous counterpart on radiography and MRI (4). In our case, plain radiographs revealed a rectangular lesion with a fuzzy image of non-uniform density in the center, when observed carefully. Although the peripheral rim was not as clear as described in previous cases, the specific features of $\mathrm{ABC}$ on MRI, including an expansile lesion surrounded by a thin lowsignal rim, increased signal with augmented T1-weighting and a lobulated contour with fluid-fluid levels were all clearly visible in our patient. The MRI presentation of our case may be described as relatively typical.

The differential diagnosis of STABC mainly includes giant cell tumor of soft tissue, giant cell tumor of the tendon sheath, extraskeletal telangiectatic osteosarcoma (EOS) and myositis ossificans.

Giant cell tumors are often associated with $\mathrm{ABC}$, since ABCs often contain a large number of giant cells. These two diseases are easily confused, and as a result it is occasionally difficult to distinguish ABC from a giant cell tumor, especially when a giant cell tumor exhibits bleeding, necrosis and cystic changes. However, giant cell tumors usually occur in adults over the age of 20 , whereas ABCs are often found in the first two decades of life. However, the results noted in Table I show that the age of patients with STABC is not fixed. Histologically, compared with giant cell tumor of soft tissue or giant cell tumor of the tendon sheath, STABC often contains more reactive bone-like tissue and woven bone with a well-defined peripheral rim, but fewer giant cells.

Differentiation between STABC and EOS is essential as they require completely different management and have different outcomes. EOS most commonly affects individuals older than 30 and is rarely encountered during the first two decades of life (17). EOS differs from STABC in that it usually has an ill-defined rim on X-rays and is more prone to becoming malignant. Microscopically, anaplastic tumor cells and atypical mitosis may be found in EOS, which do not exist in STABC.

Although radiography and CT may reveal certain similarities between STABC and myositis ossificans, i.e., a radiolucent lesion with a thin rim of ossification at the periphery, the presence of septa within the lesion in STABC may differentiate it from myositis ossificans (4). Myositis ossificans usually contain a solid inner component, whereas STABC does not show the presence of any solid parts (except for the septa). Nevertheless, STABC may also be secondary to myositis ossificans. In this case, cystic spaces filled with blood and connective tissue septa are also the main points of differentiation.

In conclusion, STABC is an extremely rare type of benign soft tissue tumor, with no more than 20 cases having been reported in the English language literature. It is occasionally secondary to lesions such as giant cell tumor, myositis ossificans and conventional osteosarcoma, therefore caution should be used in the diagnosis of STABC. If STABC is correctly diagnosed, surgical treatment should be implemented with complete excision considered the most appropriate treatment.

\section{Acknowledgements}

This study was supported by the National High Technology Research and Development Program of China (863 program) (grant no. 2009AA03Z311), the National Natural Science Foundation of China (grant no. 81071472) and the Key Disciplines of Shanghai Municipal Education Commission (grant no. J50206).

\section{References}

1. Nielsen GP, Fletcher CD, Smith MA, Rybak L and Rosenberg AE: Soft tissue aneurysmal bone cyst: a clinicopathologic study of five cases. Am J Surg Pathol 26: 64-69, 2002.

2. Rodriguez-Peralto JL, Lopez-Barea F, Sanchez-Herrera S and Atienza M: Primary aneurysmal cyst of soft tissues (extraosseous aneurysmal cyst). Am J Surg Pathol 18: 632-636, 1994.

3. Wang XL, Gielen JL, Salgado R, Delrue F and De Schepper AM: Soft tissue aneurysmal bone cyst. Skeletal Radiol 33: 477-480, 2004.

4. Ajilogba KA, Kaur H, Duncan R, McFarlane JH and Watt AJ: Extraosseous aneurysmal bone cyst in a 12-year-old girl. Pediatr Radiol 35: 1240-1242, 2005.

5. Ellison DA, Sawyer JR, Parham DM and Nicholas R Jr: Soft-tissue aneurysmal bone cyst: report of a case with $\mathrm{t}(5 ; 17)$ (q33;p13). Pediatr Dev Pathol 10: 46-49, 2007.

6. Pietschmann MF, Oliveira AM, Chou MM, et al: Aneurysmal bone cysts of soft tissue represent true neoplasms: a report of two cases. J Bone Joint Surg Am 93: e45, 2011.

7. van de Luijtgaarden AC, Veth RP, Slootweg PJ, et al: Metastatic potential of an aneurysmal bone cyst. Virchows Arch 455: 455-459, 2009.

8. Fan JY, Wang YF, Han B, Ji YR, Song HD and Fan XQ: FOXL2 mutations in Chinese families with Blepharophimosis syndrome (BPES). Transl Res 157: 48-52, 2011.

9. Jaffe HL and Lichtenstein L: Solitary unicameral bone cyst: with emphasis on the roentgen picture, the pathologic appearance and the pathogenesis. Arch Surg 44: 1004-1025, 1942.

10. Panoutsakopoulos G, Pandis N, Kyriazoglou I, Gustafson P, Mertens F and Mandahl N: Recurrent t(16;17)(q22;p13) in aneurysmal bone cysts. Genes Chromosomes Cancer 26: 265-266, 1999.

11. Sciot R, Dorfman H, Brys P, et al: Cytogenetic-morphologic correlations in aneurysmal bone cyst, giant cell tumor of bone and combined lesions. A report from the CHAMP study group. Mod Pathol 13: 1206-1210, 2000.

12. Dal Cin P, Kozakewich HP, Goumnerova L, Mankin HJ, Rosenberg AE and Fletcher JA: Variant translocations involving $16 \mathrm{q} 22$ and $17 \mathrm{p} 13$ in solid variant and extraosseous forms of aneurysmal bone cyst. Genes Chromosomes Cancer 28: 233-234, 2000.

13. Oliveira AM, Perez-Atayde AR, Inwards CY, et al: USP6 and CDH11 oncogenes identify the neoplastic cell in primary aneurysmal bone cysts and are absent in so-called secondary aneurysmal bone cysts. Am J Pathol 165: 1773-1780, 2004.

14. Ye Y, Pringle LM, Lau AW, et al: TRE17/USP6 oncogene translocated in aneurysmal bone cyst induces matrix metalloproteinase production via activation of NF-kappaB. Oncogene 29: 3619-3629, 2010.

15. Oliveira AM, Perez-Atayde AR, Dal Cin P, et al: Aneurysmal bone cyst variant translocations upregulate USP6 transcription by promoter swapping with the ZNF9, COL1A1, TRAP150, and OMD genes. Oncogene 24: 3419-3426, 2005.

16. Geiersbach K, Rector LS, Sederberg M, et al: Unknown partner for USP6 and unusual SS18 rearrangement detected by fluorescence in situ hybridization in a solid aneurysmal bone cyst. Cancer Genet 204: 195-202, 2011.

17. Lee KH, Joo JK, Kim DY, Lee JS, Choi C and Lee JH: Mesenteric extraskeletal osteosarcoma with telangiectatic features: a case report. BMC Cancer 7: 82, 2007. 